\title{
BIM 技术在国有项目管理上的应用现状分析
}

\author{
石丰文
}

济南城投建设发展有限公司, 山东济南 250000

[摘要]近些年来 BIM 技术得到了广泛的应用, 将其应用到国有项目中可以实现智能化管理并可以对传统管理过程中的不足进 行弥补, 在未来其将会成为国有项目管理中的主要技术。在使用 BIM 技术对国有项目进行管理时应充分了解 BIM 技术地特点, 并对其在国有项目中的应用现状进行分析，以此来提升其在国有项目中的应用水平。

[关键词]BIM 技术; 国有项目; 应用现状

DOI：10.33142/aem.v2i1.1419 中图分类号: F299.233.42;F270.7 文献标识码：A

\section{Analysis on the Application Status of BIM Technology in the Management of State-owned Projects}

SHI Fengwen

Jinan Urban Investment Construction Development Co., Ltd., Jinan, Shandong, 250000, China

\begin{abstract}
In recent years, BIM Technology has been widely used in state-owned projects, which can realize intelligent management and make up for the shortcomings in the traditional management process. In the future, it will become the main technology in state-owned project management. When using BIM Technology to manage state-owned projects, we should fully understand the characteristics of BIM Technology, and analyze its application status in state-owned projects, so as to improve its application level in state-owned projects.
\end{abstract}

Keywords: BIM Technology; state-owned projects; application status

\section{BIM 技术概述及特点}

\section{1 BIM 技术概述}

BIM 技术属于建筑信息模型，充分地利用了三维虚拟数字化技术，并可以应用到项目的各个阶段，实现对项目各个阶 段的系统化的管理。在国有项目中应用 BIM 技术，应将其与计算机技术进行结合，充分地利用计算机及信息技术中的优 势强化对项目各个阶段的管理, 实现管理的自动化、智能化, 以此来提升项目建设质量, 降低管理成本, 实现绿色管理。

\section{2 主要特点}

第一，可视化特点分析。国有项目中应用 BIM 技术可以实现可视化管理，可以为相关的管理人员提供更加精准的 信息数据，在对项目结构与项目特点进行分析后保证工程可以顺利开展。可以充分地利用 BIM 技术中的三维、四维虚 拟数字技术清晰的展现出项目的结构特点, 在充分了解建筑结构特点的基础上保证施工进度与施工质量。第二, 协同 性特点分析。国有项目中所涉及的内容、专业相对较多, 且参与方也比较多。因此, 要想保证国有项目顺利进行应充 分的做好各部门间的协调管理工作，并对相关的信息资源进行整合，实现信息资源共享，使各参建方可以更好的沟通。

BIM 技术中的协同性是比较明显的可以将所需要的内容进行汇总, 为项目建设提供更加可靠的数据信息。第三，模拟性 特点分析。利用 BIM 技术中的模拟特点可以对现场施工建设进行模拟, 让参建人员充分了解项目内容及其中所存在的 问题, 可以对问题进行及时的处理, 实现对项目内容的整体优化。可以将 BIM 技术对施工过程进行模拟, 对施工过程 中可能产生的问题、风险等进行预测; 根据工程实际情况制定施工方案, 为国有项目管理提供更加可靠的参考信息, 使施工方案更加完善。同时, 可以通过模拟施工对项目中所存在的问题进行合理的解决, 强化质量、成本等方面的管 理, 以此来保证项目的综合效益。

\section{BIM 技术目前在国有投资项目管理应用过程中存在的现象}

\section{1 各参建单位应用 BIM 技术进行项目管理的重视程度不一样}

国家投资建设项目的参建单位一般有建设单位、设计勘察单位、监理单位和施工单位, 有的建设项目建设单位可 能还要委托项目管理单位。在这些单位中, 应用 BIM 技术进行设计最好的单位是勘察设计单位, 但在项目建设管理中, 却没有提倡应用 BIM 技术进行项目管理的主动权; 其次是一些有实力的施工单位, 如中国建筑、中国铁建等大型央企 单位。除了国家重点建设项目或 EPC 项目建设单位要求应用 BIM 技术管理外, 在一般国有建设项目上, 建设单位、监 理单位等普遍没有 BIM 人材或组建 BIM 团队，应用 BIM 技术进行项目管理还处在意识阶段。 


\section{2 BIM 技术应用缺少投入}

我国政府一直提倡在房屋建筑、市政公用基础设施建设项目上, 要大力推广 BIM 技术应用, 有的地方政策文件也 出台了具体落实措施。但在 BIM 技术应用资金如何投入方面，无论是政策、法规、还是建设工程合同和咨询委托合同 的有关法律条文，均没有涉及到资金投入事项，致使项目建设单位在项目投资概算中很少独立考虑 BIM 技术应用费用。 因此, 一般项目设计上, 设计单位如果接收不到建设单位的明确要求或委托合同中没有专门 BIM 技术设计费用, 就按 传统设计；项目管理单位、监理单位或施工单位也就按传统项目管理或施工。

\section{3 国有项目中 BIM 技术的应用}

\section{1 利用 BIM 技术强化进度管理}

3.1.1 构建 Revit 与 Navisworks 施工模拟

在对国有项目特点及图纸进行分析后将与进度管理的相关信息输入到 BIM 系统中, 形成 BIM-4D 模型, 并将信息导 入到系统平台中。利用 Revit 软件后可以实现建模进度与施工进度的统一, 在建模过程中可以采用分区方式、分层方 式、分构件方式, 实现建筑、结构、机电及幕墙等多专业间的协同管理。管理人员在进行进度管理时可以对进度管理 情况进行实时查看并可以对工程变化情况进行动态模拟。将已构成的 Revit 软件模型导入到 Navisworks 分析软件中, 以此来对进度管理进行优化, 利用思维模型、部分专业模型对施工进度进行碰撞分析, 充分利用可视化实现进度管理, 管理人员可以更加直观的了解进度管理中的问题并对主要的影响因素进行分析, 以此来控制变更情况, 实现对进度的 有效控制。

\section{1.2 构建协同平台}

构建 BIM 系统平台的过程中可以将 WBS 作为主线, 以工作包为单位, 将 BIM 构件作为载体, 以此来实现成本、进 度、质量、安全等方面的系统化管理, 最终利用 BIM 技术实现对进度的精细化管理, 以此来提升进度管理平台化、信 息化, 通过有效的进度管理, 提升质量管理水平并降低整体成本, 提升综合效益。在 BIM 平台上各参建方可以进行实 时沟通并对施工中的问题进行有效的处理。利用 VR 技术、AR 技术等对现场具体情况进行模拟，进度管理人员在对实际 进度与原进度进行对比后可以对原有的进度管理计划进行优化, 在 BIM 平台中对施工内容进行合理的安排, 以此来满 足进度管理要求。利用 BIM 设计对整体施工进度进行控制并做好后期评价工作。可以在 BIM 平台中实现对进度的全过 程管理, 可以将其与初始模型进行比对, 然后将相关信息数据进行输出并反映出各参建方所要完成的工作内容、管理 责任，为进度管理提供便利，保证进度管理效率。

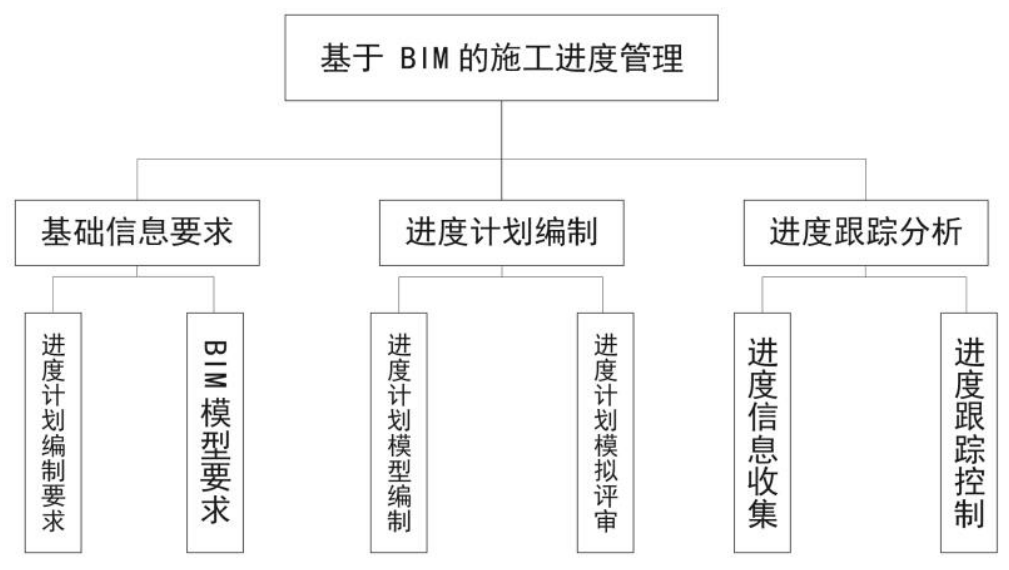

\section{2 利用 BIM 技术强化质量管理}

\subsection{1 三维现场平面布置}

在对现场情况进行分析后, 可以将与施工区域、周边环境相关的信息录入到 BIM 模型中, 构建起三维现场平面模 型, 利用此对施工质量进行控制, 为国有项目管理顺利开展奠定基础。利用 BIM 模式对施工现场进行布置, 对施工中 的各个环节进行规划, 同时对材料设备进场、出场的路线、存储方式进行布置, 设计出不同的方案, 从中选取最佳方 案，保证方案的完整性，同时可以减少返工情况的出现，在节约成本的基础上提升国有项目建设质量。

\subsection{2 碰撞检测}

碰撞检测时应用 BIM 技术可以在施工前找到不同专业在空间上的碰撞并可以及时发现图纸中的问题, 形成碰撞报 告后可以避免返工或窝工现象, 在保证工期的基础上来降低整体成本, 从而实现质量管理目标。同时, 对工程管理内 容的正确性与可行性进行验证并将其作为管理依据。由于国有项目中涉及的数据量相对较大，这样也增加了各专业碰 
建筑工程与管理・ 2020 第2卷 第 1 期

Architecture Engineering and Management.2020, 2(1)

撞检测的难度, 给设备安装、协调等带来困难, 国有项目在进行机电管线综合布置时可以利用碰撞检测对其进行优化。 通过碰撞检测可以发现更多的问题并可以利用 BIM 模型对碰撞问题进行有效的解决, 可以利用二维码对施工现场质量 管理提供指导。

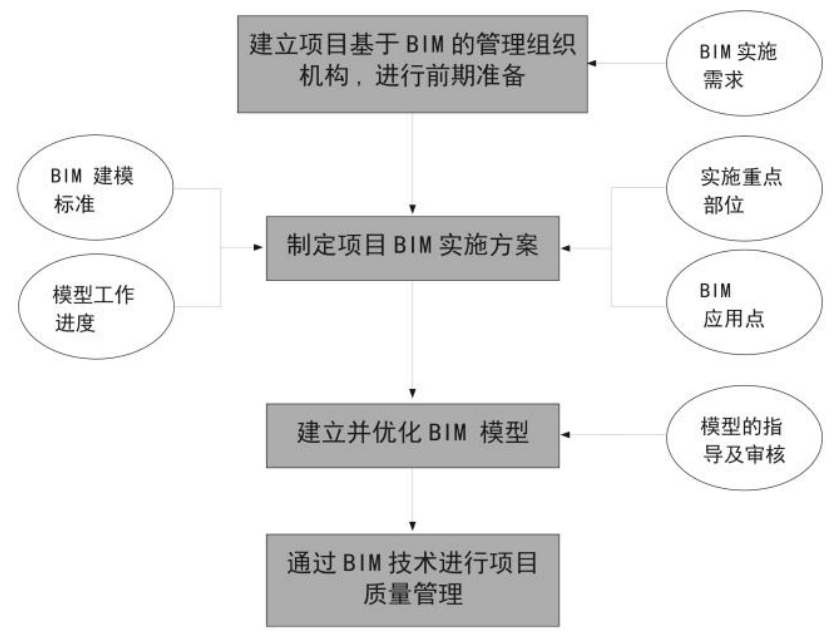

\section{3 利用 BIM 技术强化成本管理}

国有项目成本管理过程中应根据实际情况构建起 3D 模型, 再与时间、工序等情况进行结合构建成本 BIM-5D 数据 库, 将施工过程中所产生的与成本管理相关的数据输入到成本信息库中, 对成本数据进行实时统计, 利用 WBS 解构对 结构进行解构并将 WBS 单位所提供的工程量数据作为主要数据汇入到成本 BIM 中。利用 BIM 技术将施工图纸数据、材 料数据及成本数据进行整合, 实现对图纸、材料等方面成本的动态化管理, 并可以实现对成本的多维度分析, 及时的、 快速的对应对变更问题并作出有效的调整, 实现对成本的动态监管, 以此来控制施工中的返工问题, 降低整体成本, 利用 BIM 技术实现成本绿色管理目标。

\section{4 利用 BIM 技术强化安全管理}

无论是怎样的项目安全管理都是首要任务, 传统的安全管理工作中多依靠人工方式, 但是随着建设环境、建设规 模的不断变化如果只单一的依靠人工管理方式已经无法满足现代管理的需要, 给管理带来一定的局限。将 BIM 技术引 用到安全管理工作中可以对安全风险进行判断、仿真模拟、动态监管并提升安全风险应急管理方案的合理性。提升安 全管理功能，使安全管理更加细致、全面，充分的发挥出 BIM 技术在安全管理中的优势。

\section{5 满足时代发展要求，提升管理人员整体素质}

现阶段, 国有项目中管理者素质的提升是关键, 传统的管理思路、管理模式已经无法顺应时代发展的要求, 因此 国有企业应对原有的管理资源进行整合, 形成全局意识, 实现对整体项目的全面化管控。可见, 只有转变管理思路才 能转换管理方式, 实现 BIM 全周期生命管理与项目全经营周期管理的信息系统的全面结合。在管理过程中 BIM 信息库 建设不断被完善, 内容也变得更加丰富且可以实现动态化管理。利用 BIM 信息库可以提升进度管理、质量管理效率, 降低整体成本, 为企业赢得更多的经济效益。

\section{4 结语}

在国有项目管理过程中, BIM 技术是一种先进的管理技术, 在进行国有项目管理时可以将不同的管理技术进行综合, 以更好的服务于建设工程项目管理, 提高管理效益。通过实践, 要想提升 BIM 技术在国有项目中的应用效果, 建设单 位应积极主动组织各参建单位应构建起数据共享平台, 增加 BIM 技术应用投入。利用项目管理评价机制进行完善, 以 此来解决国有项目中 BIM 技术应用方面的不足, 提升进度、质量、成本及安全管理水平, 以此来保证项目整体经济效 益, ${ }^{[2]}$ 向建设项目智慧管理迈进。

\section{[参考文献]}

[1]洪长英. BIM 技术在建筑工程项目管理中的应用 $[\mathrm{J}]$. 居舍, 2019 (6) : 72 .

[2]任龙昌,刘文刚. BIM 技术在现代建筑工程项目管理中的应用研究 [J]. 建筑技术开发, 2019 (4) : 89-90.

作者简介: 石丰文 (1972.11-), 男, 毕业学校: 北京理工大学, 专业: 机械电子专业, 单位: 济南城投建设发展有限 公司，职务：工程管理，职称：工程师。 